Research Article

\title{
Prevalence and Trajectory of COVID-19-Associated Hypercoagulability Using Serial Thromboelastography in a South African Population
}

\author{
Sarah Alexandra van Blydenstein $\mathbb{D}^{1},{ }^{1}$ Colin Nigel Menezes $\mathbb{D}^{2},{ }^{2}$ Nicole Miller, ${ }^{3}$ \\ Naomi Johnson, ${ }^{3}$ Bavinash Pillay, ${ }^{3}$ Barry F. Jacobson, ${ }^{4}$ and Shahed Omar $\mathbb{1}^{5}$ \\ ${ }^{1}$ Division of Pulmonology, Department of Internal Medicine, Chris Hani Baragwanath Academic Hospital, \\ University of the Witwatersrand, Johannesburg, South Africa \\ ${ }^{2}$ Division of Infectious Diseases, Department of Internal Medicine, Chris Hani Baragwanath Academic Hospital, \\ University of the Witwatersrand, Johannesburg, South Africa \\ ${ }^{3}$ Department of Internal Medicine, Chris Hani Baragwanath Academic Hospital, University of the Witwatersrand, \\ Johannesburg, South Africa \\ ${ }^{4}$ Department of Molecular Medicine and Haematology, National Health Laboratory Service, University of the Witwatersrand, \\ Johannesburg, South Africa \\ ${ }^{5}$ Department of Critical Care, Chris Hani Baragwanath Academic Hospital, University of the Witwatersrand, \\ Johannesburg, South Africa
}

Correspondence should be addressed to Sarah Alexandra van Blydenstein; savanblydenstein@gmail.com

Received 8 October 2021; Accepted 23 November 2021; Published 22 December 2021

Academic Editor: Samuel A. Tisherman

Copyright (c) 2021 Sarah Alexandra van Blydenstein et al. This is an open access article distributed under the Creative Commons Attribution License, which permits unrestricted use, distribution, and reproduction in any medium, provided the original work is properly cited.

Introduction. The coagulation abnormalities resulting from severe acute respiratory syndrome coronavirus 2 (SARS-CoV-2) have been attributed to inflammation and subsequent cytokine storm. Thromboelastography (TEG) is a point-of-care test used to assess clot formation and degradation in whole blood and is an indicator of the overall real-time coagulopathic state of the patient. Methods. A single-centre, prospective, observational cohort study was conducted in South Africa, analysing the coagulation patterns of 41 patients with hypoxia related to SARS-CoV-2 using serial thromboelastography (TEG) on admission, after 48 hours, and at resolution of hypoxia/day 10. Results: Two-thirds $(n=26)$ were women. The median age was 61 (IQR 50-67), and the majority $(88 \%)$ were Black patients. Almost half (22) of the patients were critically ill and ventilated, with median SOFA and SAPS2 scores of 3 and 22 (IQR2-4 and 18-30), respectively. The prevalence of hypercoagulability was 0.54 (95\% CI 0.46-0.62), whilst 29/41 (0.71, CI 0.64-0.78)) met the definition of hypofibrinolysis. Differences between the hypercoagulable (HC) and nonhypercoagulable groups remained apparent at 48 hours after anticoagulation. At this time point, the $\mathrm{K}$ time was significantly lower $\left(p^{<} 0,01\right)$, and the $\alpha$-angle $\left(p^{<} 0,01\right)$ and maximum amplitude (MA) $\left(p^{<} 0,01\right)$ were significantly higher in the HC cohort. At resolution of hypoxia, or day 10, only MA was significantly higher in the hypercoagulable group compared to the non-hypercoagulable group $(p=0.01)$. The initial impairment in fibrinolysis (Ly30), $\alpha$ angle, and MA were significantly associated with mortality, with $p$ values of $0.006,0.031$, and 0.04 , respectively. Conclusions. In this South African population, hypercoagulability was a highly prevalent phenomenon in COVID-19 disease. It was typified by hypofibrinolysis and a persistently elevated MA, despite anticoagulation therapy. 


\section{Introduction}

Severe acute respiratory syndrome coronavirus 2 (SARSCoV-2) causes coronavirus disease 2019 (COVID-19), a multisystem disease, which is typified by hypoxia, inflammation, increased microthrombosis and macrothrombosis [1-4], and fibrinolysis shutdown $[5,6]$. HC has been characterised using various viscoelastic tests (VETs), specifically thromboelastography (TEG).

Severe COVID-19 is associated with coagulation abnormalities, specifically a raised D-dimer and an increased fibrinogen, which correlates with a worse prognosis [7] and an increased risk of thrombotic events [7-9], and is not consistent with a disseminated intravascular coagulopathy (DIC) [9-11] or a consumptive process. [7] The aetiology of hypercoagulability may be linked to endothelial inflammation, neutrophil extracellular traps, and increased platelet activity with increased microvesicles. [11, 12].

Currently, there is no gold standard definition of hypercoagulability as defined by TEG parameters, but $\mathrm{HC}$ is typified by a low Ly30<1\%, a MA $>69 \mathrm{~mm}$, an $\alpha$-angle of $>77^{\circ}$, R-time of $<4.3$ minutes, and a K-time of $<0.8$ minutes $[9,11,13-15]$. These findings demonstrate a decreased clot formation time, an increased clot strength, and impairment of the fibrinolytic system. Variations in the definition of $\mathrm{HC}$ include combinations of abnormalities in MA, clot formation time, and $\alpha$-angle. $[5,7,10,15,16]$ This COVID-19associated state of hypercoagulability and impaired fibrinolysis has been associated with an increased risk of thromboembolism [5, 7, 11] and thrombotic events, respectively. [6].

There have been several studies [6, 7, 10, 15-17] assessing TEG abnormalities at a single time point and even fewer at another point during COVID-19 infection. [8, 9, 11].

Using a robust definition that included one hypercoagulant/procoagulant parameter in addition to impaired lysis, we sought to determine an accurate estimate of the prevalence of COVID-19-associated hypercoagulability. In addition, we performed two further TEG assessments to describe the dynamic changes after 48 hours of anticoagulation and at resolution/day 10 of hypoxemia. To our knowledge, this is the first study in the South African population.

\section{Methods}

2.1. Study Design and Site. This was a prospective, observational, cohort study of COVID-19 adult patients in a South African hospital.

2.2. Study Population, Inclusion, and Exclusion Criteria. All patients aged 18 years and older who tested positive for the SARS-CoV-2 using polymerase chain reaction (PCR) assay and were admitted between November 2021 and March 2021 were considered for enrolment and were sequentially approached during weekday office hours. Patients were included if they were expected to survive for longer than 48 hours and met the criteria for severe disease or critical illness. Severe disease was defined as oxygen saturation $\leq 92 \%$ with a respiratory rate $\geq 25$ and, therefore, requiring supplemental oxygen support without the need for invasive or noninvasive ventilation. Critical illness was defined as hypoxemia and the need for additional ventilatory support, in the form of noninvasive or invasive ventilation. We excluded patients if they were pregnant, on oestrogen replacement therapy, and chronic anticoagulation therapy, including, but not limited to, aspirin, warfarin, clopidogrel, heparin, enoxaparin, and direct-acting oral anticoagulants.

2.3. Procedure. All enrolled patients had blood collected into a citrated tube for coagulation assessment using thromboelastography with the $\mathrm{TEG}^{\circledR} 6 \mathrm{~s}$, Haemonetics, Braintree, MA, USA, at three set time points during hospital admission:

(i) Time point 1 (day of admission, either before enoxaparin administration or at least four hours after enoxaparin administration)

(ii) Time point 2 (48 hours, three hours postenoxaparin administration)

(iii) Time point 3 (resolution of hypoxemia-off oxygen therapy, three hours postenoxaparin administration) or on day 10 of admission, whichever occurred first

Patient demographic, clinical, laboratory, and hospital survival data were extracted from clinical notes. The following scores were calculated from the clinical information: disseminated intravascular coagulation (DIC) score as per International Society on Thrombosis and Haemostasis (ISTH) criteria [18], sepsis-induced coagulopathy (SIC) score [19], simplified acute physiology score 2 (SAPS2) [20], and sequential organ failure assessment (SOFA) scores [21], at the above three time points unless the enrolled subject was discharged or demised before the defined time.

2.4. TEG ${ }^{\circledR} 6 s$ Methodology and Study Definitions [22]. The TEG is a point-of-care test, is used to assess the formation and degradation of a clot in whole blood, and demonstrates abnormalities in coagulation, which may not be apparent when using standard coagulation tests including prothrombin time (PT), activated partial thromboplastin time (aPTT), platelet number, and antithrombin. TEG ${ }^{\circledR} 6$ s assays employ various activators, including kaolin, heparinase, and tissue factor. The reaction time $(R)$ describes the time to initial fibrin formation and $2 \mathrm{~mm}$ amplitude (initiation phase) and informs about clotting factors. The kinetic $(K)$ time is the time taken to achieve a given clot strength of $20 \mathrm{~mm}$ amplitude (amplification phase) and indicates the initial phase of fibrin cross-linking. The $\alpha$-angle measures the speed of clot formation and assesses the thrombin burst (propagation phase). Both the $K$-time and $\alpha$-angle are dependent on fibrinogen. The maximum amplitude (MA) indicates the maximum strength of the clot and depends on platelet function and the contribution of fibrin. The lysis time at 30 minutes (Ly30) indicates the amount of fibrinolysis within 30 minutes of MA. [22]. 
We used a strict study definition for hypercoagulability (HC) requiring the presence of both an abnormality of lysis (low Ly30) and at least one of the following: a reduced $R$ time, reduced $K$-time, elevated $\alpha$-angle, or an elevated MA on admission.

2.5. Anticoagulation Protocol. Enoxaparin dosing was based on the COVID-19 hospital protocol. Therapeutic dosing was used when there was an increased risk of hypercoagulability based on an elevated $D$-dimer, in the face of critical illness and severe hypoxemia or in the presence of thromboembolic events. Alternatively, prophylactic enoxaparin was administered if patients were hypoxemic, had a normal $\mathrm{D}$-dimer level, and had no contraindication to enoxaparin administration. TEGs were performed at admission before anticoagulation administration and, at time points 2 and 3, were performed 3-4 hours after the anticoagulation dose to standardise the effect of anticoagulation and measure antifactor 10 a. All patients received appropriate anticoagulation as per hospital protocol.

2.6. Outcome Measures. We applied the study definition of $\mathrm{HC}$ to the patient data to calculate the prevalence of HC. We described the trajectory of TEG parameters using data from admission, after 48 hours, and at resolution. We used the admission non-TEG characteristics to describe the hypercoagulable and non-hypercoagulable states. Lastly, we described the TEG and non-TEG characteristics and their association with mortality.

2.7. Statistical Analysis. Study data were collected and managed using REDCap ${ }^{\circledR}$ (Research Electronic Data Capture) electronic data capture tool hosted at the University of the Witwatersrand. Statistical analyses were performed using Statistica ${ }^{\circledR}$ version 13.3 (TIBCO Software Inc., USA). Continuous variables are expressed as median (interquartile range (IQR)), and proportions/percentages were used for categorical variables. Continuous data were compared using the Mann-Whitney $U$ test, whilst proportions were compared using the chi-square test. A $p$-value $<0.05$ was considered statistically significant. We based our sample size on the minimum number of 12 required per group as a rule of thumb for a pilot study. [23] Assuming a mortality rate of $40 \%$, we required a minimum sample size of 20 per group.

2.8. Ethics Considerations. Approval was received from the University Human Research Ethics Committee (Medical), M200728. Written informed consent from the patient or patient surrogate was obtained as per local ethics committee guidelines.

\section{Results}

3.1. Demographics. A total of 41 patients with COVID-19 were included from 4 November 2020 and 23 March 2021. All patients (41) had a TEG on admission, 38 had a second (at 48 hours after admission), and 25 had a third (either on day ten if still hypoxemic or at the resolution of hypoxemia, whichever came first). Two-thirds $(n=26,63 \%)$ were women. The median age was 61 years (IQR 50-67). The majority (88\%) were Black patients. The median SAPS II score was 22 (IQR 18-30).

Almost half $(22 / 41,54 \%)$ of the patients were critically ill and ventilated, and 19 were classified as "severe disease" (not ventilated but hypoxic and requiring supplemental oxygen therapy). On the day of admission, the median SOFA score was 3 (IQR 2-4), ISTH DIC score was 2 (IQR 0-2), and median SIC score was 2 (IQR 2-3). Half (54\%) of enrolled patients had coexistent hypertension, $41 \%$ had diabetes mellitus, 39\% had human immunodeficiency virus (HIV), and $15 \%$ were obese.

Overall, 20 of 39 (51\%) patients were placed on therapeutic anticoagulation, and 19 of 39 (49\%) were placed on prophylactic doses of enoxaparin. There were no differences in the proportion of patients on therapeutic anticoagulation between the HC and non-HC group, $X[2]=0.24, p=0.62$.

3.2. Prevalence. Twenty-two of the $41(54 \%)$ patients met the study definition of hypercoagulability (HC) with a prevalence of 0.54 (95\% CI $0.46-0.62$ ), whilst $32 / 41$ (prevalence of 0.78 , CI $0.72-0.85$ ) met the definition of hypofibrinolysis with Ly $30<1 \%$. Using a Ly 30 threshold of $\leq 0.1 \%$, the prevalence was 0.71 (CI $0.64-0.78$ ).

3.3. Trajectory of TEG Parameters from Admission to Resolution. Hypercoagulability was defined by admission (T1) TEG parameters. Differences between the hypercoagulable and non-hypercoagulable groups become more apparent at 48 hours implying that there is a progression of the HC state, which only starts resolving after at least 48 hours. Although the $\alpha$-angle and MA trended higher at baseline, these differences did not reach a significant statistical difference until 48 hours later. At 48 hours, the K-time is significantly lower $\left(p^{<} 0.01\right.$ the $\alpha$-angle $\left(p^{<} 0.01\right)$ and MA $\left(p^{\ulcorner} 0.01\right)$ is significantly higher in the HC cohort. At resolution of hypoxia, or day 10 , only MA was significantly and persistently higher in the hypercoagulable group compared to the non-hypercoagulable group $(p=0.01)$. As shown in Table 1 and Figure 1, this difference was driven by survivors (80\%). At both 48 hours and resolution, median anti-Xa levels were within the range for anticoagulation prophylaxis, $0.42(0.21-0.52)$ and $0.4(0.27-0.84)$, respectively, within the HC group, and $0.42(0.21-0.59)$ and $0.37(0.26-0.7)$ in the non-HC group.

3.4. Hypercoagulable and Non-Hypercoagulable Groups. As shown in Table 2, platelet count at admission was significantly higher in the hypercoagulable group. There was also a trend to a lower haemoglobin level in the HC.

3.5. Mortality. Admission SOFA score, SAPS 2 score, and LDH were significantly higher amongst nonsurvivors. The initial impairment in fibrinolysis (Ly30), $\alpha$-angle, and MA were significantly associated with mortality. As shown in 
TABLE 1: Changes in the TEG and anti-Xa levels between admission and at day 10/resolution.

\begin{tabular}{|c|c|c|c|c|c|c|}
\hline \multirow{2}{*}{ TEG CKH } & \multicolumn{2}{|r|}{$T 1$} & \multicolumn{2}{|r|}{$T 2$} & \multicolumn{2}{|r|}{ T3 } \\
\hline & $n$ & Median (IQR) & $\mathrm{n}$ & Median (IQR) & $n$ & Median (IQR) \\
\hline \multicolumn{7}{|l|}{ Hypercoagulable } \\
\hline LY30-CK (\%) & 22 & $0[0-0.1]$ & 20 & $0[0-0]$ & 16 & $0[0-0]$ \\
\hline TEG-ACT (sec] & 22 & 83.2 [78.5-87.9] & 20 & 92.6 [78.5-106.6] & 16 & $92.6[73.9-106.6]$ \\
\hline$R$-time & 22 & $4.6[4-5.2]$ & 20 & $5[4.6-6.2]$ & 16 & $4.5[3.7-6]$ \\
\hline$K$-time & 22 & $0.8[0.8-1.0]$ & 20 & $0.8[0.8-0.9]^{*}$ & 16 & $0.8[0.8-1.1]$ \\
\hline$\alpha$ angle & 22 & $78.2[76.9-79]$ & 20 & $79.4[77.6-81.1]^{*}$ & 16 & $77.5[75.6-79.7]$ \\
\hline MA & 22 & $69.5[68.8-70.4]$ & 20 & $70.3[68.7-71.8]^{*}$ & 16 & $70[68.9-72.3]^{*}$ \\
\hline \multicolumn{7}{|l|}{ Non-hypercoagulable } \\
\hline LY30-CK (\%] & 19 & $0[0-0]$ & 17 & $0[0-0]$ & 9 & $0[0-0]$ \\
\hline TEG-ACT (sec] & 19 & $97.3[78.5-116]$ & 18 & $97.3[87.9-116]$ & 9 & 97.3 [87.9-106.6] \\
\hline$R$-time & 19 & $4.6[3.5-5.3]$ & 18 & $5.4[4.9-6.1]$ & 9 & $4.7[4.3-5.9]$ \\
\hline$K$-time & 19 & $1[0.8-1.8]$ & 18 & $1.3[1-1.6]^{*}$ & 9 & $1[0.8-1.3]$ \\
\hline$\alpha$-Angle & 19 & $75.4[69.9-77.5]$ & 18 & $72.8[71.5-76.9]^{*}$ & 9 & $77.1[72.7-79.7]$ \\
\hline MA & 19 & 65.3 [53.4-68.9] & 18 & $64[58-65.7]^{*}$ & 9 & $65.6[61.6-68.6]^{*}$ \\
\hline
\end{tabular}

${ }^{*}$ Statistically significant. Data are expressed as median (interquartile range). T1 (admission), T2 (48 hours), T3 (resolution of hypoxia/day 10), TEG thromboelastography, ACT activated clotting time, CKH citrated kaolin heparinase, $R$ reaction, $\mathrm{K}$ kinetics, MA maximum amplitude, LY30 lysis at 30 minutes.

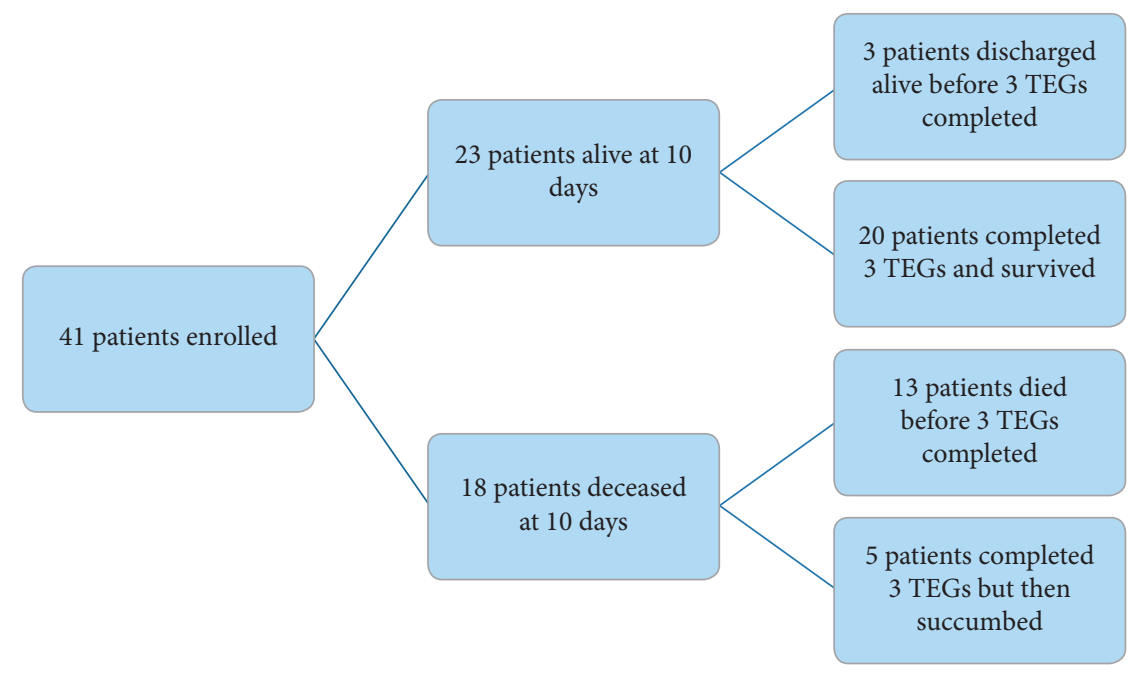

FIGURE 1: Flow diagram of a number of TEGs completed.

Table 3, there was a trend to a higher D-dimer value amongst nonsurvivors.

\section{Discussion}

Severe COVID-19 is associated with coagulation abnormalities, known as CAC, specifically a raised D-dimer, which correlates with a worse prognosis [7] and an increased risk of thrombotic events. [14] The coagulation abnormalities are considered to be a hypercoagulable state typified by increased fibrinogen and increased D-dimers [7-9] and are not consistent with a disseminated intravascular coagulopathy (DIC) [9-11] or a consumptive process. [7] The aetiology of hypercoagulability may be linked to endothelial inflammation, neutrophil extracellular traps, and increased platelet activity with increased microvesicles. [11, 12].

Whilst traditional coagulation tests have been useful in the measurement of CAC, TEG is a point-of-care test used to assess the clot formation and degradation in whole blood in real time and can identify the individual contributions of the endothelium, the platelets, and the clotting factors. The fibrinolysis shutdown, described by Tsantes et al. [15] as maximum Ly30<3.5\%, cannot be appreciated using conventional coagulation parameters. There have been several studies [15] looking at predominantly single-time point TEG assessments in critically ill patients with COVID-19, with some [8] having a repeat TEG, but for the first time, a third TEG assay was performed in this study of serial TEGs in patients with COVID-19, at time of resolution of hypoxia or day ten of illness.

The main finding was that half of the study population (54\%) met the study definition of hypercoagulable state on their initial TEG, and $71 \%$ had a Ly30 $\leq 0.1 \%$. No patients met the ISTH DIC criteria, with a mean DIC score of 2 , and a mean SIC score of 2 . The range of $\mathrm{HC}$ documented in studies has been variable. Considering clot lysis time alone as a 
TABLE 2: Non-TEG characteristics.

\begin{tabular}{|c|c|c|c|c|}
\hline Variables & All patients, $n=41$ & HC, $n=22$ & Non-HC, $n=19$ & $p$ value \\
\hline Age (year) & $61[50-67]$ & $62.5[51-68]$ & $56[46-64]$ & 0.22 \\
\hline Female, $n(\%)$ & $26(63)$ & $14 / 42(34 \%)$ & $12 / 41(29 \%)$ & \\
\hline \multicolumn{5}{|l|}{ Blood parameters } \\
\hline Haemoglobin (g/dL) & $13.2[11.6-14.2]$ & $12.05[10.6-14.00]$ & $13.3[13.00-15.00]$ & 0.06 \\
\hline $\mathrm{LDH}(\mathrm{U} / \mathrm{L})$ & $752[489-901]$ & 759 [495-842] & 651 [489-989] & 0.98 \\
\hline D-dimers (mg/L) & $0.585[0.38-1.39]$ & $0.58[0.38-1.0]$ & $0.96[0.39-2.12]$ & 0.45 \\
\hline Fibrinogen $(\mathrm{mg} / \mathrm{dL})$ & $6.7[5.6-7.8]$ & $6.7[6-7.9]$ & $6.5[4.9-7.3]$ & 0.40 \\
\hline Platelets $\left(\times 10^{9} / \mathrm{L}\right)$ & 249 [195-292] & $265[237-342]$ & $221[167-280]$ & $0.02^{*}$ \\
\hline $\mathrm{PT}(\mathrm{sec})$ & $13.45[13.0-14.4]$ & $13.9[13.0-14.4]$ & 13.4 [12.9-14.5] & 0.75 \\
\hline aPTT (sec) & $25.6[22.35-30.7]$ & 24.7 [21.9-28.7] & $25.85[23.2-32.0]$ & 0.60 \\
\hline INR & $1.11[1.06-1.19]$ & $1.13[1.06-1.18]$ & $1.11[1.06-1.2]$ & 0.90 \\
\hline Enoxaparin, $\mathrm{n}=\mathbf{3 9}$ & $n=39$ & $n=21$ & $n=18$ & 0.62 \\
\hline Therapeutic, $n(\%)$ & $20(51)$ & $10(48)$ & $10(56)$ & \\
\hline Prophylactic $n(\%)$ & $19(49)$ & $11(52)$ & $8(44)$ & \\
\hline \multicolumn{5}{|l|}{ Severity } \\
\hline Severe & $19(46)$ & $10(53)$ & $9(47)$ & \\
\hline Critically ill & $22(54)$ & $12(55)$ & $10(45)$ & 0.9 \\
\hline SOFA & $3[2-4]$ & $3[2-4]$ & $2[2-5]$ & 0.49 \\
\hline SAPS2 & $22[18-30]$ & $26[21-31]$ & $21[18-27]$ & 0.13 \\
\hline DIC & $2[0-2]$ & $2[0-2]$ & $2[0-3]$ & 0.16 \\
\hline SIC & $2[2-3]$ & $2[2-3]$ & $2[2-3]$ & 0.87 \\
\hline Mortality, n (\%) & $18(43.9)$ & $8(44.4)$ & $10(55.6)$ & 0.30 \\
\hline Resolution of hypoxemia by day $10, \mathrm{n}(\%)$ & $13(32)$ & $8(36)$ & $5(26)$ & 0.49 \\
\hline
\end{tabular}

TABLE 3: Factors associated with mortality.

\begin{tabular}{|c|c|c|c|c|c|}
\hline \multirow{2}{*}{ Initial variable } & \multicolumn{2}{|c|}{ Survivors } & \multicolumn{2}{|c|}{ Nonsurvivors } & \multirow{2}{*}{$p$-value } \\
\hline & Valid $n$ & Median (IQR) & Valid n & Median (IQR) & \\
\hline SOFA score & 23 & $2[2-3]$ & 18 & $4[2-6]$ & 0.017 \\
\hline SAPS2 score & 23 & $20[18-26]$ & 18 & $28[21-43]$ & $0.006^{*}$ \\
\hline DIC score & 23 & $2[0-2]$ & 18 & $2[0-3]$ & 0.112 \\
\hline SIC score & 23 & $2[2-3]$ & 18 & $2[2-3]$ & 0.490 \\
\hline Platelets $x 10 * 9 / \mathrm{L}$ & 23 & 269 [244-328] & 18 & 196 [167-264] & $0.004^{*}$ \\
\hline Haemoglobin g/dL & 23 & $13.3[11.6-14.2]$ & 18 & $13.2[11.3-14.3]$ & 0.765 \\
\hline $\mathrm{LDH} \mathrm{u} / \mathrm{L}$ & 15 & 513 [413-757] & 12 & $889[387-1051]$ & $0.003^{*}$ \\
\hline Fibrinogen $\mathrm{mg} / \mathrm{dL}$ & 15 & $6.7[6.0-7.5]$ & 12 & $6.5[5.35-7.95]$ & 0.980 \\
\hline D-dimers mg/L & 21 & $0.56[0.34-0.97]$ & 17 & $0.96[0.39-2.12]$ & 0.089 \\
\hline INR & 22 & $1.11[1.05-1.19]$ & 17 & $1.13[1.07-1.18]$ & 0.440 \\
\hline PT sec & 21 & $13.4[12.8-14.2]$ & 17 & $13.5[13.1-14.4]$ & 0.601 \\
\hline aPTT sec & 21 & $25.2[21.9-26.5]$ & 15 & $29.9[22.8-32.6]$ & 0.141 \\
\hline LY30-CK (\%) & 23 & $0.1[0.00-2.00]$ & 18 & $0.0[0.0-0.0]$ & $0.006^{*}$ \\
\hline $\mathrm{R}-\mathrm{CKH}$ & 23 & $4.9[4.2-5.5]$ & 18 & $4.0[3.4-5.2]$ & 0.138 \\
\hline $\mathrm{K}-\mathrm{CKH}$ & 23 & $0.8[0.8-1.0]$ & 18 & $1.0[0.8-1.8]$ & 0.081 \\
\hline Ang-CKH & 23 & $78.2[75.3-79.6]$ & 18 & 75.95 [69.90-78.10] & $0.031^{*}$ \\
\hline MA-CKH & 23 & $69.1[66.5-70.4]$ & 18 & $68.3[53.4-69.4]$ & $0.040^{*}$ \\
\hline
\end{tabular}

*Statistically significant. Data are expressed as median (interquartile range). LDH: lactate dehydrogenase, PT: prothrombin time, aPTT: activated partial thromboplastin time, INR: international normalised ratio, SOFA: sequential organ failure assessment, SAPS2: simplified acute physiology score 2, DIC: disseminated intravascular coagulopathy, SIC: sepsis-induced coagulopathy, TEG: thromboelastography, CKH: citrated kaolin heparinase, $R$ : reaction, K: kinetics, MA: maximum amplitude, LY30: lysis at 30 minutes, Ang: angle, Anti-Xa: antifactor Xa.

marker for hypofibrinolysis, Bocci et al. found a Ly30 time of $0 \%$ on initial TEG in all $40(100 \%)$ of their cohort [8], whilst Blasi et al. described "no lysis" in only $13 \%$ of the COVID-19 cohort. [10] A recent systematic review found that all the eligible studies had a Ly $30<1 \%$ and three studies had a Ly30 of $0 \%$. This study used a threshold for Ly30 of less than or equal to $0.1 \%$, and our data showed a high prevalence $(71 \%)$ of hypofibrinolysis [14].

Hypofibrinolysis on its own is an insufficient marker of abnormal coagulation, and at least one other TEG marker 
indicating increased clot burden may be found in $20 \%$ of "healthy" individuals. [24] To ensure robustness, our definition of hypercoagulability required both a lysis abnormality and one other TEG abnormality. The prevalence of COVID-19-associated HC ranges from 30\% [16] (considering either an increased MA, K-time, or $\alpha$-angle) to $74 \%$ [25] when only a MA $>69 \mathrm{~mm}$ is considered. Our proposed definition yields a prevalence of $54 \%$, which may be less prone to overestimation and underestimation.

Aside from the absence of a gold standard definition, the timing of the viscoelastic test (VET) is likely to impact prevalence estimates. This is evident with studies conducting VET up to two weeks from admission. $[26,27]$ We chose to define HC based on admission TEG parameters to avoid the effects of anticoagulation. There may be additional heterogeneity based on different commercially available VET manufacturers (ROTEM, TEG, Quantra, and ClotPro) and differences in the patient population. Whilst our study provides the first data from South Africa in a predominantly Black population (88\%), the effect of different analysers requires further investigation.

Despite similar anticoagulation strategies with similar proportional utilisation of prophylactic and therapeutic anticoagulation in the two groups, the HC group showed a significantly greater persistence of a functional procoagulant state over time. This is demonstrated with the hypercoagulable group demonstrating a shorter amplification (K-time) and greater propagation (higher $\alpha$-angle) at 48 hours compared to the non-hypercoagulable group. There are no other data that we could find in the literature to compare these findings with. Both these processes normalise compared to the non-HC group by day 10/resolution. Two factors can be postulated to contribute to this change. The first is likely the effect of the administered anticoagulant, and the second may be the improvement of the HC state with disease resolution over time. After the administration of heparin in both groups, there was a nonstatistical increase in $R$-time between admission (preheparin) and 48 hours after admission ( 3 hours after the fourth dose of heparin). It is difficult to interpret the effect of heparin on the $R$-time as we used a heparinase-corrected reading, which may attenuate the increase in $R$-time.

The evolution of the functional coagulation variable relating to maximal clot strength (MA) is of great interest. The MA was significantly elevated and greater in the $\mathrm{HC}$ group when compared to the non-HC group. This elevation was present at 48 hours and persisted to resolution and/or day 10. This was found despite similar anticoagulation strategies and efficacy (anti-Xa levels). This difference was driven by the findings in the survivors $(80 \%$ of those with TEGs at T3). Bocci et al. [8] described a similar persistence of TEG abnormalities at seven days despite therapeutic-dose anticoagulation.

Notably, $80 \%$ of clot strength and integrity are dependent on platelet function. [22] Our intervention included full anticoagulation for roughly half of the hypercoagulable group, whilst Bocci et al. used full anticoagulation for the entire group. The delayed persistence of this elevation may indicate that even therapeutic anticoagulation may not be able to completely reverse this hypercoagulable state.
Consideration should be given to the role of platelet aggregation, platelet hyperactivity, and microthrombus formation as these may be a neglected part of the pathophysiologic process of severe COVID-19. [28, 29] The MA represents the eventual strength of the fibrin clot and is not only predominantly dependent on platelet count in nonCOVID-19 patients, interacting via GPIIb/IIIa, but also on fibrin. Fibrin has been thought to be the dominant role player in clot strength in patients with COVID-19. [30] The persistently elevated MA despite the resolution of hypoxia results in the continued stimulation of platelet activity in the damaged endothelium, particularly of the lungs, and the role of antiplatelet therapy may require further investigation within this cohort of patients.

The mechanism of platelet abnormality is multifactorial. There are HC and endothelialitis that occur with SARS-Co$\mathrm{V}-2$ infection, exacerbated by hypoxia (itself a platelet activator), and the milieu becomes procoagulant and proinflammatory. [2] Neutrophil extracellular traps may also play a role in initiating coagulation [2], as may an autoimmune component to the thrombocytopenia triggered by SARS-Co$\mathrm{V}$-2, or direct infection of the haemopoietic cells, as was seen in SARS, a similar coronavirus.

There have also been studies showing a normal [9] or increased platelet count [11], and possible causes include hypoxia with stimulation of hypoxia-inducible factor and increased megakaryocyte activity within the lung and bone marrow. [31] Furthermore, the spike protein of SARS-Co-V2 can directly stimulate platelets. [32] Our own findings suggest an association between a higher platelet count and this state.

Hranjec et al. used platelet mapping and VET to guide the treatment of patients with COVID-19, and they corrected the platelet-induced aspect of the coagulopathy with either aspirin or clopidogrel or both (in addition to heparin), which was associated with improved outcomes. Viecca et al. performed a proof-of-concept case-control study and showed that patients treated with acetylsalicylic acid and clopidogrel had a reduction in alveolar to arterial oxygen gradient, consistent over seven days, with a concomitant increase in $\mathrm{PaO} 2 / \mathrm{FiO} 2$ ratio, with no bleeding incidences. [33].

The fibrinolysis shutdown (Ly30) in our study was significantly lower in the nonsurvivors. This has been previously described, with Wright et al. [6] demonstrating a correlation between fibrinolysis shutdown and thrombotic events, and Bocci et al. [8] demonstrated a worse 28-day outcome within the group who had Ly30 $<1 \%$ and an elevated $\mathrm{D}$-dimer, and an association between $\mathrm{HC}$ and rate of thrombotic events (TEs) and renal failure $[6,11,34]$ amongst patients with COVID-19.

The median $\alpha$-angle (clot propagation) was approximately $76^{\circ}$ in nonsurvivors and $78^{\circ}$ in survivors. Although this was statistically different, it is unlikely that these are clinically different as they both hover around the upper reference limit and the coefficient of variation of the assay is certainly larger than this difference. A similar relationship between MA and mortality is present. Both survivors and nonsurvivors have an elevated MA, but the absolute 
difference $(0.8 \mathrm{~mm})$ is unlikely to be clinically important. It is likely that we should, therefore, consider hypercoagulability as a continuum of effect, and larger studies may be required to establish clinical thresholds.

The combination of hypofibrinolysis and persistently increased MA alludes to the as yet not fully understood COVID-19 coagulopathy but suggests that platelets should not be overlooked and speaks to the complex nature of the disease process.

4.1. Limitations. This study was limited by its observational nature. Furthermore, it was a small study, at a single centre, and thromboembolic events were not assessed.

\section{Conclusions}

In this South African population, hypercoagulability is a highly prevalent phenomenon in COVID-19 disease. It is typified by hypofibrinolysis and a persistently elevated MA, despite anticoagulation therapy.

\section{List of abbreviations used}

anti-Xa: Anti-factor $X$ a

aPTT: Activated partial thromboplastin time

CAC: $\quad$ COVID-19-associated coagulopathy

CHBAH Chris Hani Baragwanath Academic Hospital

SOP: $\quad$ Standard Operating Procedure

CI: $\quad$ Confidence interval

CKH: $\quad$ Citrated kaolin heparinase

COVID-19: Coronavirus disease 2019

DIC: Disseminated intravascular coagulation

HC: Hypercoagulability

IQR: Interquartile range

ISTH DIC: International Society on Thrombosis and Haemostasis Disseminated Intravascular Coagulation score

K-time: $\quad$ Kinetic time

LDH: $\quad$ Lactate dehydrogenase

Ly(30): $\quad$ Lysis time at 30 minutes

MA: $\quad$ Maximal amplitude

PT: $\quad$ Prothrombin time

R-time: Reaction time

SARS-CoV- Severe acute respiratory syndrome

2: $\quad$ coronavirus 2

SAPS2: $\quad$ Simplified acute physiology score 2

SD: $\quad$ Standard deviation

SIC: $\quad$ Sepsis-induced coagulopathy

SOFA: Sequential organ failure assessment

TE: Thrombotic events

TEG: Thromboelastography

TTP: Thrombotic thrombocytopenic purpura

VET: Viscoelastic test.

\section{Data Availability}

Anonymised supporting data will be made available upon request in an Excel spreadsheet.

\section{Ethical Approval}

Approval was received from the University Human Research Ethics Committee (Medical), M200728.

\section{Consent}

Written informed consent from the patient or patient surrogate was obtained as per local ethics committee guidelines.

\section{Disclosure}

Qiagen had no role in the study design, collection, analysis, and interpretation of data, in writing of the report, or in the decision to submit the article for publication.

\section{Conflicts of Interest}

No author has any conflict of interest to declare.

\section{Authors' Contributions}

SAVB contributed to the conception, design of the work, data and sample collection, and interpretation of data, drafted the work, substantively revised it, approved the submitted version, and agreed both to be personally accountable for the author's own contributions and to ensure that questions related to the accuracy or integrity of any part of the work, even ones in which the author was not personally involved, are appropriately investigated, resolved, and the resolution documented in the literature. CNM contributed to the conception and design of the work, substantively revised it, approved the submitted version, and agreed both to be personally accountable for the author's own contributions and to ensure that questions related to the accuracy or integrity of any part of the work, even ones in which the author was not personally involved, are appropriately investigated, resolved, and the resolution documented in the literature. NM contributed to data collection and sample collection, substantively revised it, approved the submitted version, and agreed both to be personally accountable for the author's own contributions and to ensure that questions related to the accuracy or integrity of any part of the work, even ones in which the author was not personally involved, are appropriately investigated, resolved, and the resolution documented in the literature. NJ contributed to data collection and sample collection, substantively revised it, approved the submitted version, and agreed both to be personally accountable for the author's own contributions and to ensure that questions related to the accuracy or integrity of any part of the work, even ones in which the author was not personally involved, are appropriately investigated, resolved, and the resolution documented in the literature. BP contributed to data collection and sample collection, substantively revised it, approved the submitted version, and agreed both to be personally accountable for the author's own contributions and to ensure that questions related to the accuracy or integrity of any part of the work, even ones in which the author was not 
personally involved, are appropriately investigated, resolved, and the resolution documented in the literature. BFJ contributed to the conception and design of the work, substantively revised it, approved the submitted version, and agreed both to be personally accountable for the author's own contributions and to ensure that questions related to the accuracy or integrity of any part of the work, even ones in which the author was not personally involved, are appropriately investigated, resolved, and the resolution documented in the literature. SO contributed to the conception, design of the work, analysis, and interpretation of data, substantively revised it, approved the submitted version, and agreed both to be personally accountable for the author's own contributions and to ensure that questions related to the accuracy or integrity of any part of the work, even ones in which the author was not personally involved, are appropriately investigated, resolved, and the resolution documented in the literature.

\section{Acknowledgments}

TEG ${ }^{\circledR} 6$ s cartridges were provided by Qiagen.

\section{References}

[1] F. A. Klok, M. J. H. A. Kruip, N. J. M. van der Meer et al., "Incidence of thrombotic complications in critically ill ICU patients with COVID-19," Thrombosis Research, vol. 191, pp. 145-147, 2020.

[2] B. S. Joly, V. Siguret, and A. Veyradier, "Understanding pathophysiology of hemostasis disorders in critically ill patients with COVID-19," Intensive Care Medicine, vol. 46, no. 8, pp. 1603-1606, 2020.

[3] J. Liu, S. Li, J. Liu et al., "Longitudinal characteristics of lymphocyte responses and cytokine profiles in the peripheral blood of SARS-CoV-2 infected patients," EBioMedicine, vol. 55, Article ID 102763, 2020.

[4] M. Ackermann, S. E. Verleden, M. Kuehnel et al., "Pulmonary vascular endothelialitis, thrombosis, and angiogenesis in covid-19," New England Journal of Medicine, vol. 383, no. 2, pp. 120-128, 2020.

[5] V. Pavoni, L. Gianesello, M. Pazzi, C. Stera, T. Meconi, and F. C. Frigieri, "Evaluation of coagulation function by rotation thromboelastometry in critically ill patients with severe COVID-19 pneumonia," Journal of Thrombosis and Thrombolysis, vol. 50, no. 2, pp. 281-286, 2020.

[6] F. L. Wright, T. O. Vogler, E. E. Moore et al., "Fibrinolysis shutdown correlation with thromboembolic events in severe COVID-19 infection," Journal of the American College of Surgeons, vol. 231, no. 2, pp. 193-203, 2020.

[7] L. Spiezia, A. Boscolo, F. Poletto et al., "COVID-19-Related severe hypercoagulability in patients admitted to intensive care unit for acute respiratory failure," Thrombosis \& Haemostasis, vol. 120, no. 06, pp. 998-1000, 2020.

[8] M. G. Bocci, R. Maviglia, L. M. Consalvo et al., "Thromboelastography clot strength profiles and effect of systemic anticoagulation in COVID-19 acute respiratory distress syndrome: a prospective, observational study," European Review for Medical and Pharmacological Sciences, vol. 24, no. 23, pp. 12466-12479, 2020.

[9] P.-Y. Cordier, C. Pierrou, A. Noel et al., "Complex and prolonged hypercoagulability in coronavirus disease 2019 intensive care unit patients: a thromboelastographic study," Australian Critical Care, vol. 34, no. 2, pp. 160-166, 2021.

[10] A. Blasi, F. A. Meijenfeldt, J. Adelmeijer et al., "In vitro hypercoagulability and ongoing in vivo activation of coagulation and fibrinolysis in COVID-19 patients on anticoagulation," Journal of Thrombosis and Haemostasis, vol. 18, no. 10, pp. 2646-2653, 2020.

[11] M. Panigada, N. Bottino, P. Tagliabue et al., "Hypercoagulability of COVID-19 patients in intensive care unit: a report of thromboelastography findings and other parameters of hemostasis," Journal of Thrombosis and Haemostasis, vol. 18, no. 7, pp. 1738-1742, 2020.

[12] M. E. Colling and Y. Kanthi, "COVID-19-associated coagulopathy: an exploration of mechanisms," Vascular Medicine, vol. 25, no. 5, pp. 471-478, 2020.

[13] N. Salem, B. Atallah, W. S. El Nekidy, Z. G. Sadik, W. M. Park, and J. Mallat, "Thromboelastography findings in critically ill COVID-19 patients," Journal of Thrombosis and Thrombolysis, vol. 51, no. 4, pp. 961-965, 2020.

[14] J. Hartmann, A. Ergang, D. Mason, and J. D. Dias, “The role of TEG analysis in patients with COVID-19-associated coagulopathy: a systematic review," Diagnostics, vol. 11, no. 2, p. 172, 2021.

[15] A. E. Tsantes, A. G. Tsantes, S. I. Kokoris et al., "COVID-19 infection-related coagulopathy and viscoelastic methods: a paradigm for their clinical utility in critical illness," Diagnostics, vol. 10, no. 10, p. 817, 2020.

[16] N. Salem, B. Atallah, W. S. El Nekidy, Z. G. Sadik, W. M. Park, and J. Mallat, "Thromboelastography findings in critically ill COVID-19 patients," Journal of Thrombosis and Thrombolysis, vol. 51, no. 4, pp. 961-965, 2021.

[17] C. Ibañez, J. Perdomo, A. Calvo et al., "High D dimers and low global fibrinolysis coexist in COVID19 patients: what is going on in there?" Journal of Thrombosis and Thrombolysis, vol. 51, no. 2, pp. 308-312, 2021.

[18] M. Levi, C. H. Toh, J. Thachil, and H. G. Watson, "Guidelines for the diagnosis and management of disseminated intravascular coagulation," British Journal of Haematology, vol. 145, no. 1, pp. 24-33, 2009.

[19] T. Iba, M. D. Nisio, J. H. Levy, N. Kitamura, and J. Thachil, "New criteria for sepsis-induced coagulopathy (SIC) following the revised sepsis definition: a retrospective analysis of a nationwide survey," BMJ open, vol. 7, no. 9, Article ID e017046, 2017.

[20] J. R. Le Gall, S. Lemeshow, and F. Saulnier, "A new Simplified Acute Physiology Score (SAPS II) based on a European/North American multicenter study," Journal of the American Medical Association: The Journal of the American Medical Association, vol. 270, no. 24, pp. 2957-2963, 1993.

[21] J.-L. Vincent, A. de Mendonca, F. Cantraine et al., "Use of the SOFA score to assess the incidence of organ dysfunction/ failure in intensive care units. Working group on "sepsisrelated problems" of the European Society of Intensive Care Medicine," Critical Care Medicine, vol. 26, no. 11, pp. 1793-1800, 1998.

[22] P. D. Tyler, L. M. Yang, S. B. Snider, A. B. Lerner, W. C. Aird, and N. I. Shapiro, "New uses for thromboelastography and other forms of viscoelastic monitoring in the emergency department: a narrative review," Annals of Emergency Medicine, vol. 77, no. 3, pp. 357-366, 2021.

[23] S. A. Julious, "Sample size of 12 per group rule of thumb for a pilot study," Pharmaceutical Statistics, vol. 4, no. 4, pp. 287-291, 2005. 
[24] R. Gui, X. Huang, M. Zhou et al., "Establishment of the normal reference range of thrombelastogram among the healthy population and pregnants in China," Iranian Journal of Public Health (English ed.), vol. 48, no. 5, pp. 841-848, 2019.

[25] K. Stattin, M. Lipcsey, H. Andersson et al., "Inadequate prophylactic effect of low-molecular weight heparin in critically ill COVID-19 patients," Journal of Critical Care, vol. 60, pp. 249-252, 2020.

[26] B. E. Fan, Y. W. Chia, C. L. L. Sum et al., "Global haemostatic tests in rapid diagnosis and management of COVID-19 associated coagulopathy in acute limb ischaemia," Journal of Thrombosis and Thrombolysis, vol. 50, no. 2, pp. 292-297, 2020.

[27] J. van der Linden, L. Almskog, and A. Liliequist, "Thromboembolism, hypercoagulopathy, and antiphospholipid antibodies in critically ill coronavirus disease 2019 patients: a before and after study of enhanced anticoagulation," Critical care explorations, vol. 2, 2020.

[28] B. K. Manne, F. Denorme, E. A. Middleton et al., "Platelet gene expression and function in patients with COVID-19," Blood, vol. 136, no. 11, pp. 1317-1329, 2020.

[29] G. Lippi, F. Sanchis-Gomar, E. J. Favaloro, C. J. Lavie, and B. M. Henry, "Coronavirus disease 2019-associated coagulopathy," Mayo Clinic Proceedings, vol. 96, no. 1, pp. 203-217, 2021.

[30] E. A. Vlot, E. J. Van den Dool, C. M. Hackeng, M. Sohne, P. G. Noordzij, and E. P. A. Van Dongen, "Anti Xa activity after high dose LMWH thrombosis prophylaxis in covid 19 patients at the intensive care unit," Thrombosis Research, vol. 196, pp. 1-3, 2020.

[31] L. Roncati, G. Ligabue, V. Nasillo et al., "A proof of evidence supporting abnormal immunothrombosis in severe COVID19: naked megakaryocyte nuclei increase in the bone marrow and lungs of critically ill patients," Platelets, vol. 31 , no. 8 , pp. 1085-1089, 2020.

[32] S. Zhang, Y. Liu, X. Wang et al., "SARS-CoV-2 binds platelet ACE2 to enhance thrombosis in COVID-19," Journal of Hematology \& Oncology, vol. 13, p. 120, 2020.

[33] M. Viecca, D. Radovanovic, and G. B. Forleo, "Enhanced platelet inhibition treatment improves hypoxemia in patients with severe Covid-19 and hypercoagulability. A case control, proof of concept study," Pharmacological Research, 2020.

[34] J. R. Mortus, S. E. Manek, L. S. Brubaker et al., "Thromboelastographic results and hypercoagulability syndrome in patients with coronavirus disease 2019 who are critically ill," JAMA network open, vol. 3, no. 6, Article ID e2011192, 2020. 